\title{
DIAZEPAM PARENTERAL NO TRATAMENTO DE EPILEPSIAS GRAVES
}

\author{
José Geraldo Speciali * \\ MICHEL PIERRE LISON **
}

No presente trabalho relatamos nossa experiência com o emprêgo de diazepam parenteral no tratamento de epilepsias rebeldes às várias associações de anticonvulsivantes usados por via oral.

\section{MÉTODOS E CASUASTICA}

Nossa casuística consta de 9 pacientes. Todos recebiam ou haviam recebido várias associaçōes de anticonvulsivantes e diversos tipos de derivados benzodiazepinicos por via oral (VO) e apresentavam, no mínimo, três crises epilépticas por semana. As medicaçoes recebidas foi acrescentado o diazepam (Valium) por via endovenosa (EV), na dose média de $1 \mathrm{mg} / \mathrm{kg}$ ( $2-3 \mathrm{mg} / \mathrm{minuto})$ para os pacientes com pêso inferior a $20 \mathrm{~kg} \mathrm{e}$, na dose de $20 \mathrm{mg}$, para os que tivessem pêso superior, dividida em duas aplicações diárias, por tempo não inferior a uma semana. Eventualmente, administrou-se a medicação por via intramuscular (IM) após, pelo menos, 6 aplicaçōes EV.

Os eletrencefalogramas (EEG) foram feitos, em aparêlho Grass, modêlo VI, de 8 canais, com eletrodos colocados segundo a posição recomendada pela Federação Internacional de Eletrencefalografia e a Neurofisiologia Clinica ${ }^{6}$. Os tracados foram obtidos em estado de vigilia ou durante o sono induzido por secobarbital. $O$ primeiro EEG foi realizado 24 horas antes do inicio da terapêutica EV. Iniciada a medicação o exame foi repetido cada dois dias e, após sua interrupção, quinzenalmente.

Os dados clínicos e eletrencefalográficos referentes a êsses pacientes estāo indicados no quadro 1 . Usamos a nomenclatura recomendada pela Liga Internacional contra a Epilepsia ${ }^{2}$. Os pacientes permaneceram internados durante os primeiros 10 dias.

O quadro 2 mostra a duração, a via de administração e a dosagem do diazepam.

Considerou-se resultado ótimo quando a redução de freqüência das crises foi maior que $75 \%$; bom, quando entre $50 \%$ e $75 \%$; mau, quando menor que $50 \%$.

\section{R E S U L T A D O S}

Obtiveram-se resultados iniciais ótimos em três pacientes (casos 4, 6 e 9); bons, em dois (casos 7 e 8) e maus em 4. Quando a melhora não surgiu nos primeiros dias, não ocorreu ulteriormente, mesmo mantendo as aplicações por mais tempo.

Departamento de Neuropsiquiatria e Psicologia Médica (Prof. J. Armbrust-Figueiredo) da Faculdade de Medicina de Ribeirão Prêto: * Médico-Residente; ** Professor de Disciplina. 


\begin{tabular}{|c|c|c|c|c|c|}
\hline Caso & Sexo & $\begin{array}{l}\text { Idade } \\
\text { (anos) }\end{array}$ & Tipos de crises & $\begin{array}{l}\text { Freqüência } \\
\text { aproximada das } \\
\text { crises }\end{array}$ & EEG \\
\hline 1 & $\mathbf{M}$ & 9 & Mioclônico-atônicas & $30 /$ dia & $\begin{array}{l}\text { Depressão da atividade de fundo à esquerda. } \\
\text { Complexos ponta-ondas agudas difusas. }\end{array}$ \\
\hline 2 & $\mathbf{F}$ & 9 & & 2/dia & $\begin{array}{l}\text { Complexos poliponta-ondas lentas ou ponta- } \\
\text { ondas lentas difusos. }\end{array}$ \\
\hline 3 & $\mathbf{M}$ & 5 & $\begin{array}{l}\text { Tônicas } \\
\text { Atônicas }\end{array}$ & $\begin{array}{l}2-3 / \text { dia } \\
7-8 / \text { dia }\end{array}$ & $\begin{array}{l}\text { Complexos poliponta-ondas lentas e ondas len- } \\
\text { tas difusos. }\end{array}$ \\
\hline 4 & $\mathbf{F}$ & 3 & $\begin{array}{l}\text { Atônicas } \\
\text { Hemi-clônicas } \\
\text { Tônico-clônicas }\end{array}$ & $\begin{array}{r}3 / \text { dia } \\
1 / \text { dia } \\
2-3 / \text { semana }\end{array}$ & $\begin{array}{l}\text { Complexos ponta-ondas lentas e ondas lentas } \\
\text { difusos. }\end{array}$ \\
\hline 5 & $\mathbf{M}$ & 5 & Mioclônico-atônicas & $3-4 /$ dia & Complexos ponta-ondas agudas difusos. \\
\hline 6 & $\mathbf{M}$ & 23 & $\begin{array}{l}\text { Versivas } \\
\text { Versivas com generalização }\end{array}$ & $\begin{array}{r}2-3 / \text { semana } \\
1 / \text { semana }\end{array}$ & $\begin{array}{l}\text { Ondas lentas fronto-temporais esquerdas com } \\
\text { repercussão para áreas homólogas. }\end{array}$ \\
\hline 7 & $\mathbf{M}$ & 32 & Ausências mioclônicas & $60-80 /$ dia & Complexos ponta-ondas e ondas lentas difusos. \\
\hline 8 & $\mathbf{F}$ & 13 & Hemitônicas & $2-3 /$ dia & $\begin{array}{l}\text { Ondas agudas e complexos onda aguda-onda } \\
\text { lenta difusos, predominando em áreas poste- } \\
\text { riores do hemisfério cerebral direito. }\end{array}$ \\
\hline 9 & $\mathbf{M}$ & 2 & $\begin{array}{l}\text { Hemitônicas } \\
\text { Atônicas }\end{array}$ & $\begin{array}{l}5-6 / \text { dia } \\
1-2 / \text { dia }\end{array}$ & Complexos ponta-ondas lentas difusos. \\
\hline
\end{tabular}

Quadro 1 - Dados clinicos e eletrencefalográficos dos 9 pacientes antes da administracão parenteral de diazepam. 


\begin{tabular}{|c|c|c|}
\hline Caso & $\begin{array}{c}\text { Duração da administração } \\
\text { parenteral (dias) }\end{array}$ & Dose $/ 24$ horas \\
\hline 1 & $\begin{array}{l}3(\mathrm{EV}) \\
6(\mathrm{IM})\end{array}$ & $\begin{array}{l}20 \\
20\end{array}$ \\
\hline 2 & $13(\mathrm{EV})$ & 10 \\
\hline 3 & $18(\mathrm{EV})$ & 10 \\
\hline 4 & $\begin{aligned} 5 & (\mathrm{EV}) \\
35 & (\mathrm{IM})\end{aligned}$ & $\begin{array}{l}10 \\
10\end{array}$ \\
\hline 5 & $\begin{array}{ll}3 & (\mathrm{EV}) \\
7 & (\mathrm{IM})\end{array}$ & $\begin{array}{l}10 \\
20\end{array}$ \\
\hline 6 & $33(\mathrm{EV})$ & 20 \\
\hline 7 & $\begin{array}{ll}3 & (\mathrm{EV}) \\
9 & (\mathrm{EV})\end{array}$ & $\begin{array}{l}20 \\
30\end{array}$ \\
\hline 8 & $\begin{aligned} 11 & (\mathrm{EV}) \\
9 & (\mathrm{IM})\end{aligned}$ & $\begin{array}{l}20 \\
20\end{array}$ \\
\hline 9 & $\begin{array}{ll}3 & (\mathrm{EV}) \\
4 & (\mathrm{IM})\end{array}$ & $\begin{array}{l}10 \\
10\end{array}$ \\
\hline
\end{tabular}

Quadro 2 - Duração, via de administração e dose, em miligramos, de diazepam, nos 9 pacientes. Legenda: $E V=$ endovenoso; $I M=$ intramuscular.

Os seguimentos clínico e EEG longitudinais dos pacientes com resultados iniciais ótimos estão indicados no quadro 3 . No caso 4 as manifestaçôes epilépticas observadas antes do tratamento nāo recidivaram; contudo, surgiram crises tônicas generalizadas em flexão. No caso 6 apareceram crises tônicas em extensão, de 5 a 10 segundos de duração, a maioria sem queda ao solo, sem recidiva das anteriores ao planejamento terapêutico. No caso 9 observaram-se crises de palidez com parada respiratória e hipotonia. O número e a intensidade das crises permaneceram aquém daqueles observados antes do seguimento.

Um dos pacientes (caso 4) começou a sentar-se, a engatinhar e melhorou seu relacionamento com os familiares e outro (caso 9) começou a ficar em pé, quando apoiado, a falar monossilabos e a apresentar melhor relacionamento social.

O seguimento EEG mostrou aumento da freqüência da atividade de fundo $e$ aparecimento de pontas difusas (caso 4, figs. 1 e 2) ou localizadas (caso 6, figs. 3 e 4) e ondas lentas focais (caso 9, figs. 5 e 6).

Os dois pacientes com bons resultados não mantiveram a melhora após a interrupção da administração parenteral.

Os principais efeitos colaterais foram sonolência discreta em três pacientes e, em um, tonturas que desapareceram após a interrupção das administraçōes parenterais.

\section{O M E N T A R I O S}

Os derivados benzodiazepinicos administrados por via EV têm sido eficazes no contrôle do estado de mal tônico-clônico generalizado ${ }^{7,10,14}$. Quando administrados por VO, resultados favoráveis foram obtidos em pacientes 


\begin{tabular}{|c|c|c|c|c|}
\hline \multirow{2}{*}{ Caso } & \multirow{2}{*}{$\begin{array}{l}\text { Freqüência } \\
\text { aproximada e } \\
\text { caracteristicas } \\
\text { das crises }\end{array}$} & \multicolumn{2}{|r|}{ E E G } & \multirow{2}{*}{$\begin{array}{c}\text { Tempo } \\
\text { de } \\
\text { segui- } \\
\text { mento } \\
\text { (meses) }\end{array}$} \\
\hline & & $\begin{array}{c}\text { Durante tratamento } \\
\text { parenteral }\end{array}$ & Após a interrupção & \\
\hline 4 & $\begin{array}{l}\text { Crises tônicas gene- } \\
\text { ralizadas em flexão } \\
(1 / \text { dia })\end{array}$ & $\begin{array}{l}\text { Diminuição dos sur- } \\
\text { tos de onda lenta. } \\
\text { Aparecimento de pon- } \\
\text { tas ritmicas difusas. }\end{array}$ & Inalteradas. & 9 \\
\hline 6 & $\begin{array}{l}\text { Crises tônicas em } \\
\text { extensão rápidas ( } 1- \\
2 / \text { semana), dois me- } \\
\text { ses após a interrup- } \\
\text { cāo da medicação } \\
\text { parenteral }\end{array}$ & $\begin{array}{l}\text { Desaparecimento dos } \\
\text { surtos de onda len- } \\
\text { ta. }\end{array}$ & $\begin{array}{l}\text { Aparecimento de pon- } \\
\text { tas de } 14 \mathrm{c} / \mathrm{seg} \text { no he- } \\
\text { misfério cerebral es- } \\
\text { querdo predominando } \\
\text { na região temporal, } \\
\text { seguidas por ondas } \\
\text { lentas. }\end{array}$ & $4 *$ \\
\hline 9 & $\begin{array}{l}\text { Crises vegetativas } \\
(1-2 / \text { mês })\end{array}$ & $\begin{array}{l}\text { Ritmos de } 16 \text { c/seg } \\
\text { e raros complexos } \\
\text { ponta-onda rápida } \\
\text { difusos. }\end{array}$ & $\begin{array}{l}\text { Aparecimento de on- } \\
\text { da lenta fronto-tem- } \\
\text { poral direita. }\end{array}$ & 6 \\
\hline
\end{tabular}

Quadro 3 - Evolução clinica e eletrencefalográfica dos três pacientes com resultados ótimos. * Com a introdução de Ro 5-402S após 4 meses houve contrôle das crises por mais 6 meses de seguimento.

com sindromes de West e de Lennox $3,4,8,9,11,13,18$, epilepsias mioclônicas progressivas $^{1,8}$ e epilepsia parcial com sintomatologia psicomotora ${ }^{17}$; contudo, foi relatada a incidência de crises tônicas ou tônico-clônicas generalizadas 4, 11, 14. Tais fatos parecem decorrer de estado reacional determinado pela maior ou menor subitaneidade com que a droga atinge o sistema nervoso central e pelo relativo predomínio de ação sôbre os mecanismos inibidores anti-recrutantes ${ }^{8}$.

Os derivados benzodiazepinicos, administrados por via parenteral, agem de modo mais intenso e duradouro sôbre a atividade propagada que sôbre a atividade inicial do foco epileptógeno ${ }^{5,12,14}$. Assim é razoável supor que a administração diária dessas drogas, por via parenteral, atenue a hiperexcitabilidade neuronal difusa existente em pacientes com epilepsias graves resultando maior eficácia dos anticonvulsivantes usuais após a interrupção do esquema terapêutico. A melhora clínica e EEG apresentada por três pacientes confirma, a nosso ver, tal hipótese.

Não houve relação entre melhora e tipo de crises, porém resultados considerados como ótimos foram obtidos em pacientes com predomínio de alterações EEG lentas (figs. 1, 3 e 5). Após a interrupção das administrações parenterais surgiram crises tônicas e, nos registros EEG, pontas rítmicas, difusas ou localizadas (figs. 2 e 4). Esses resultados demonstram a dificuldade em controlar pacientes com tenđência à sincronização EEG rápida. 


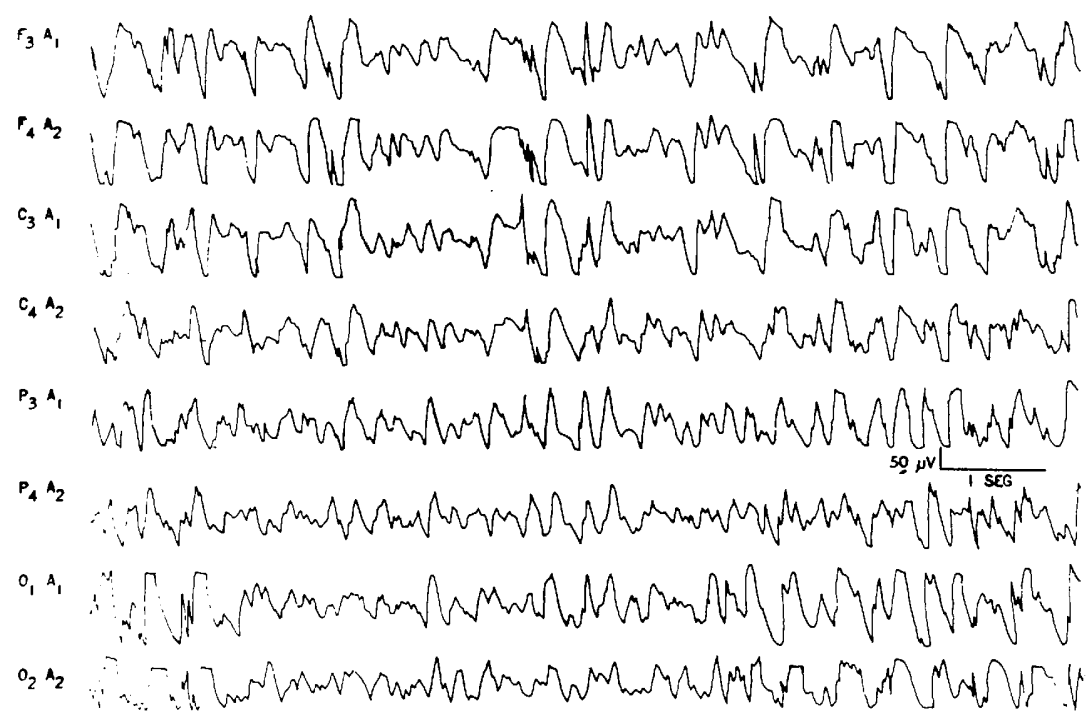

Fig. 1 - Caso 4. Sono barbitúrico. Antes do tratamento.

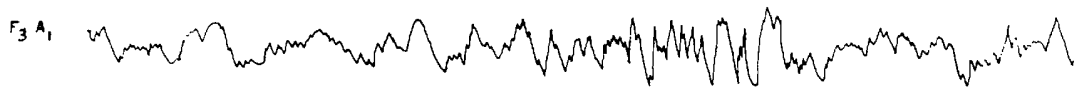

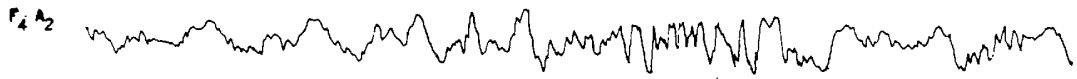

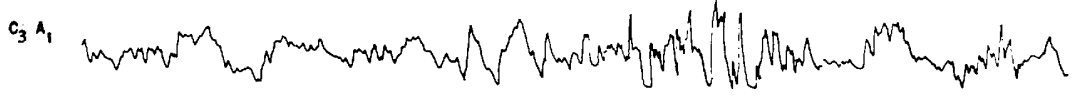

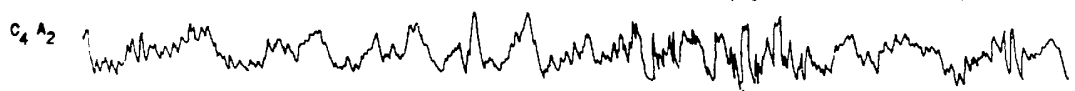

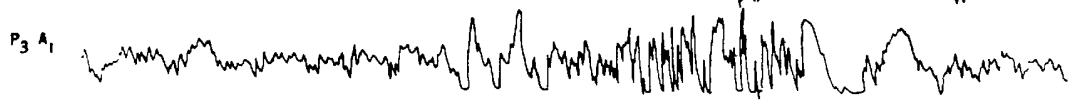

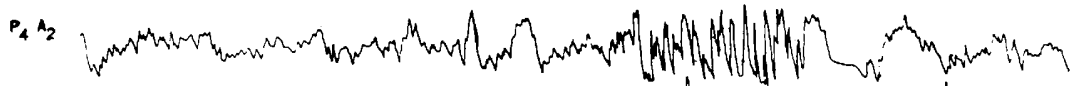

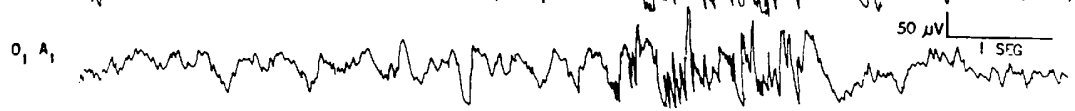

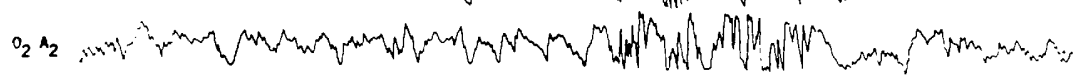

Fig. 2 - Caso 4. Sono barbitürico. Durante o tratamento. Aumento de freqüência da atividade de fundo, diminuicão dos surtos de ondas lentas e aparecimento de pontas difusas. 


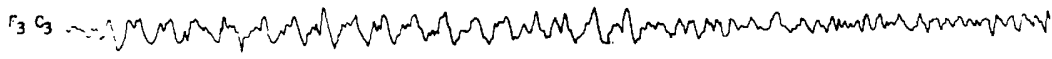

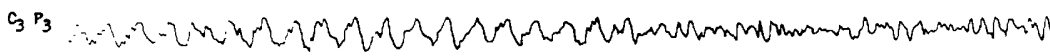

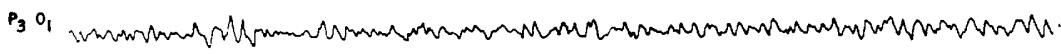

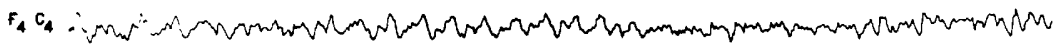

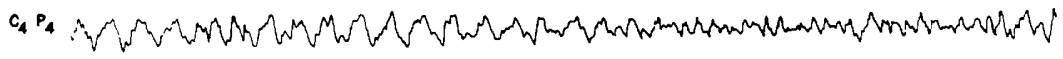

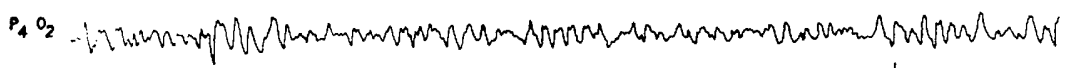

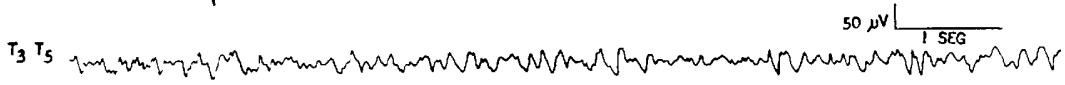

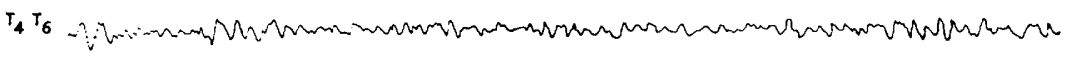

Fig. 3 - Caso 6. Vigilia. Antes do tratamento. Ondas lentas fronto-temporais esquerdas com repercussão para areas homólogas.

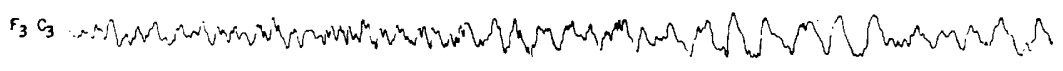

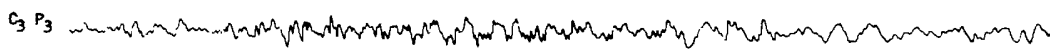

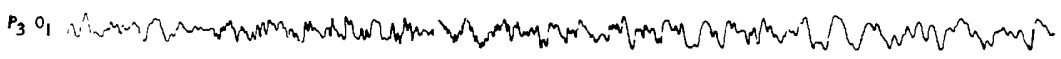

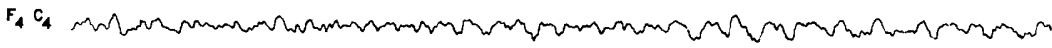

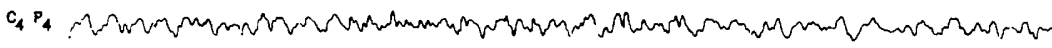

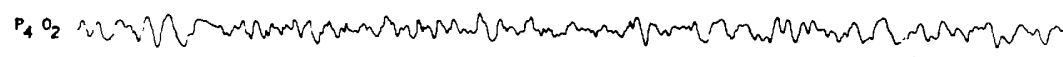

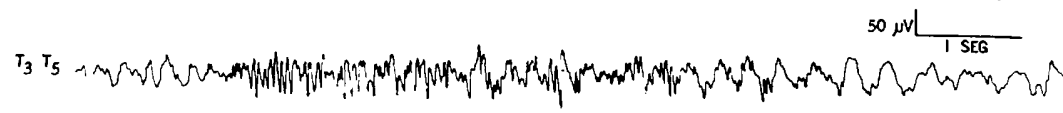

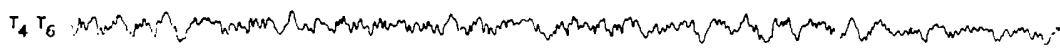

Fig. 4 - Caso 6. Após a interrupção da administração parenteral de diazepam. Dois minutos de hiperpnéia. Pontas rítmicas de $14 \mathrm{c} / \mathrm{seg}$ de projeção no hemisfério cerebral esquerdo predominando em areas temporais, seguidas por ondas lentas, sem manifestações clinicas. 


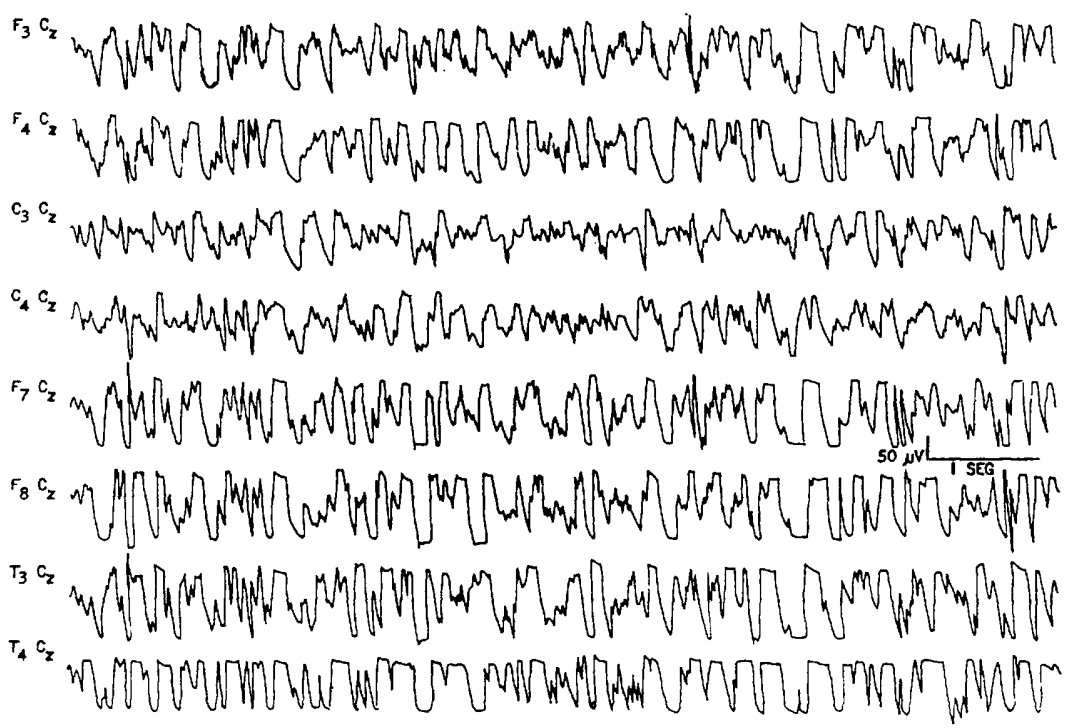

Fig. 5 - Caso 9. Sono barbitúrico. Antes do tratamento.

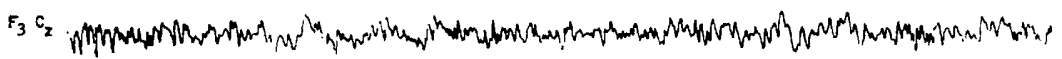

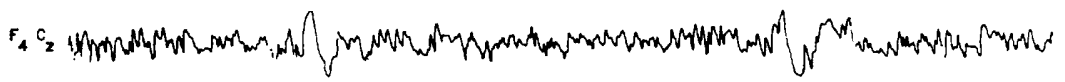

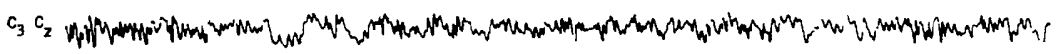

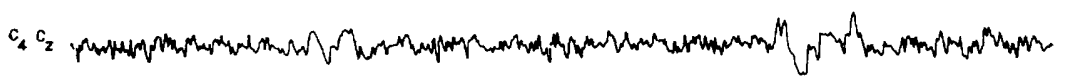

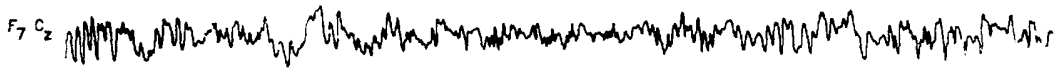

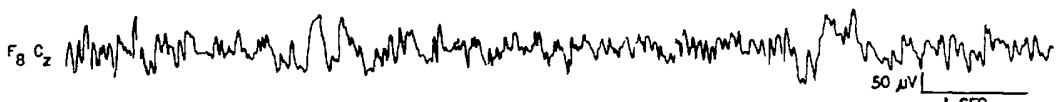

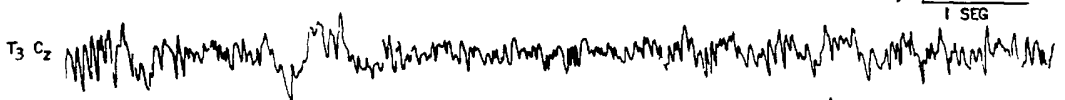

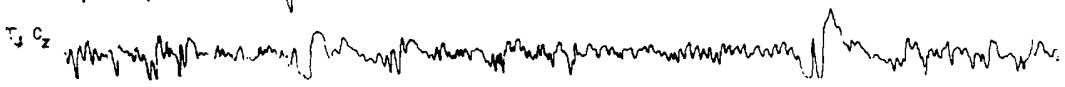

Fig. 6 - Caso 9. Sono barbitúrico. Após a interrupção da administração parenteral de diazepam. Onda lenta fronto-temporal direita. 
Recentemente Mutani e col. ${ }^{16}$ não conseguiram controlar estado de mal tônico em paciente de 65 anos com dessincronização EEG (descarga reticular subtalâmica) pela administração IM de Luminal e EV de Valium.

A maior incidência atual de crises tônicas, inclusive em adolescentes e adultos, parece estar relacionada com o uso de novos esquemas terapêticos. Tal fato já tinha sido observado, em 1963, por Miribel e col. ${ }^{15}$. A introdução dos derivados benzodiazepínicos no tratamento, em particular, das encefalopatias epilépticas da infância acentuou essa tendência ${ }^{8}$. Observações pessoais, não publicadas, demonstram a incidência de fenômenos semelhantes em crianças com síndrome de Lennox tratadas com o sal sódico do ácido din-propilacético (Depakine).

As manifestações epilépticas tônicas de provável origem talâmica mediana (ritmos recrutantes bissincronos), extra-talâmica mediana (ritmos recrutantes localizados) e reticular subtalâmica (dessincronização) constituem difícil problema terapêutico.

\section{RESUMO E CONCLUSOES}

As modificaçōes dos quadros clinico e EEG foram estudadas em 9 pacientes com manifestações epilépticas rebeldes às medicações anticonvulsivantes habituais, quando submetidos à administração parenteral diária de diazepam (Valium) e após sua interrupção. Houve diminuição do número e da duração das crises, superior a $75 \%$, em três pacientes. Esses resultados são satisfatórios, considerando a gravidade dos quadros epilépticos e foram obtidos em pacientes com predomínio de alterações EEG lentas antes de iniciar o esquema terapêutico. Nāo se verificaram efeitos colaterais relevantes na época da administração parenteral. Em dois pacientes foi observado o aparecimento de crises tônicas coincidindo com o aumento de elementos EEG paroxisticos rápidos, localizados ou difusos.

\section{S U M M A R Y}

\section{Treatment of severe epilepsies with parenteral diazepam}

Changes of the clinical pictures and electroencephalographic patterns were studied in 9 patients suffering from epileptic seizures non responsive to common anticonvulsivants, when submitted to daily parenteral administration of diazepam (Valium) and after its interruption. There was decrease of the number and duration of seizures, over to $75 \%$, in 3 patients. These results can be considered as satisfatory, considering the intensity of the seizures. The best results were obtained in those patients which showed predominance of slow waves in the EEGs prior to the begin of the treatment. No side effects were observed during the treatment. Two patients developed tonic seizures coincident with the increasing of fast paroxistic EEG patterns, of localized or diffuse type. 
$R$ E F E R N C I A

1. DECLERCK, A. - Diagnostic et traitement de l'épilepsie myoclonique progressive type Unverricht-Lundborg. Acta neurol. Belg. 68:471, 1968.

2. GASTAUT, H. - Clinical and electroencephalographic classification of epileptic seizures. Epilepsia (Amsterdam) 11:104, 1970.

3. GASTAUT, H.; ROGER, J.; LOB, H. \& TASSINARI, C. A. - L'action du diazepam (Valium) dans le traitement des formes non convulsives de l'épilepsie géneralisée (absences et états d'absences de types divers). Sem. Hôp. Paris 41:1541, 1965.

4. GIBBS, F. A. \& ANDERSON, E. M. - Treatment of hypsarhythmia and infantile spasms with a Librium analogue. Neurology (Minneapolis) 15:1173, 1965.

5. HERNANDEZ-PEON, R.; RINCON, J. T. \& CHAVEZ, G. I. - Estudio clinico, electromiográfico y electroencefalografico sobre las acciones relajante y convulsiva del Ro-5-2807. Neurol. Neurocir. Psiquiat. (México) 3:174, 1962.

6. JASPER, H. H. - The ten-twenty electrode system of the international federation. Electroencephal. Clin. Neurophysiol. 10:371, 1958.

7. LEFEVRE, A. B. \& GAZAL, S. - Tratamento do estado de mal epiléptico em crianças pelo Valium. Arq. Neuro-Psiquiat. (São Paulo) 26:155, 1968.

8. LISON, M. P. - Efeitos dos derivados benzodiazepinicos na epilepsia. Estudos eletrencefalográficos longitudinais. Arq. Neuro-Psiquiat. (São Paulo) 28:247, 1970.

9. LISON, M. P. \& FASSONI, L. F. - Estudo clínico-eletrencefalográfico Iongitudinal em pacientes epilépticos tratados com Ro 5-4023. Arq. Neuro-Psiquiat. (São Paulo) 28:25, 1970.

10. LISON, M. P. \& FASSONI, L. F. - Emprêgo do Mogadon endovenoso em pacientes epilépticos. Estudo clínico e eletrencefalográfico. Arq. Neuro-Psiquiat. (São Paulo) 26:351, 1968.

11. LISON, M. P. \& MEGA, D. - Espasmos em flexão: estudo clínico e eletrencefalográfico de pacientes tratados com Ro-4-5360. Arq. Neuro-Psíquiat. (São Paulo) 25:87, 1967.

12. LOMBROSO, C. T. - Treatment of status epilepticus with diazepam. Neurology (Minneapolis) 16:629, 1966.

13. MARKHAM, C. H. - The treatment of myoclonic seizures of infancy and childhood with LA-1. Pediatrics 34:511, 1964.

14. MEGA, D. \& LISON, M. P. - Valium endovenoso no tratamento do estado de mal epiléptico. Arq. Neuro-Psiquiat. (São Paulo) 26:145, 1968.

15. MIRIBEL, J.; FAVEL, P.; NIETO, M.; GÉRIN, P. \& COURJON, J. - Situation nosologique des crises toniques axiales dans le cadre de l'épilepsie. A propos de 18 observations. Rev. Neurol. (Paris) 109:324, 1963.

16. MUTANI, R.; BERGAMINI, R. \& FARIELLO, R. - A case of status epilepticus with tonic expression (so called "Reticular epilepsy"): physiopathological study. Epilepsia (Amsterdam) 11:321, 1970.

17. OLLER-DAURELLA, L. - El Valium y el Mogadon en el tratamiento de los automatismos. Rev. Esp. Oto-Neuro-Oftamol. y Neurocir. 24:233, 1965.

18. OLler-DAURELla, L. - Sindrome de Lennox. Expaxes, Barcelona, 1967.

Departamento de Neuropsiquiatria e Psicologia Médica - Faculadade de Medicina - Ribeirão Prêto, SP - Brasil. 